\title{
Digital Technologies and Elaboration of Mechanisms for Expert Examination of Scientific and Technical Potential of Universities
}

\author{
Ksenia E. Amelina ${ }^{1 *}$, Vladimir A. Minaev ${ }^{1}$, and Alexander V. Shchepkin ${ }^{2}$ \\ ${ }^{1}$ Bauman Moscow State Technical University, 2nd Baumanskaya str., 5/1, 105005, Moscow, Russia \\ ${ }^{2}$ V.A. Trapeznikov ICS of RAS, Moscow, Russia
}

\begin{abstract}
Within the framework of using digital technologies, this article substantiates mechanisms for examining scientific and technical potential of universities with introduction of methods of the active system management. Rigorous mathematical proofs are provided of the equilibrium state existence in expert examination procedures reflecting the experts' subjectivity. In particular, experts' interest in the examination results, as well as in their own personal ratings are demonstrated. It is concluded that digitalization of scientific and educational activities in modern universities possesses an important consequence in the form of elaborating programs for optimal management of their intellectual activity results. In addition, considered mechanisms of expert examination procedures make it possible with a high degree of correctness to evaluate complex projects connected to digitalization of scientific and technical products and optimization of resource management in executing programs at the universities.
\end{abstract}

\section{Introduction}

Any person is living in the modern information society, where social, cultural and economic conditions of life are more and more influenced by information, level of its implementation and accessibility. Knowledge accumulation is progressing at the accelerating pace. This process could be called really global without any exaggeration.

Integration of traditional approaches with opportunities provided by digital environment in temporary conditions is the only correct vector in developing scientific and educational activities of higher education institutions (HIE). It is not sufficient in the world of new opportunities just to be able to use digital technologies; it is necessary to understand the ways to increase effectiveness and efficiency of professional activities as a result of using information technologies. It is required to find new ways to improve efficiency of both educational, scientific and technical work of universities through the use of digital technologies. One of the key areas here is development and formation of the university

* Corresponding author: amelina@bmstu.ru 
scientific and technical potential. Stable mechanisms in managing this potential and effective protection of the intellectual property rights contribute to the results of intellectual activity and increasing its intensity, which is the basis for modern economy in the developed countries.

Technical University is a model successfully combining two aspects of using the principles of intellectual property management in the educational process and in the practical activity. Modern services improve the quality of scientific, technical and innovation activities infrastructure transferring it to the digital format, and, as a result, increasing the speed of performing the required actions, enhancing accessible complexity of tasks being solved, etc. Artificial intelligence potential is still to be revealed; and analytical processing of big data and using new technologies are to gain momentum.

As of today, unified methods for assessing effectiveness of scientific and innovation projects and programs in universities were not developed, which is largely due to complexity of systematic description of such an object as scientific activity in the HEI environment and ambiguity of concepts used in assessing scientific and educational characteristics of modern Russian HEIs and their intellectual property.

Expert examination mechanisms appear to be an effective systematic tool in assessing implementation of scientific and technical projects and programs at universities, their development potential; and this is the way of playing various scenarios in the course of scientific, research and innovation work in higher education institutions.

\section{Mechanisms in expert examination of university development potential}

Expert examination mechanisms are an integral part of all mechanisms in generating scientific and technical potential of universities. Works devoted to studying the expert examination mechanisms [1-3] note that it is necessary to take into account the fact that the experts themselves are either interested in the results of the expert examination, or in increasing their own ratings, which depends, on how often during the expert examination their assessment coincides with the resulting assessment by all the experts. Let us show, how to take into account this kind of interest ensuring correctness of expert assessments in modern university projects and programs. To do this, let us implement rigorous mathematical proofs of the corresponding equilibrium state existence in the expert procedures.

Let us accept the following notations:

$N=\{1,2, \ldots, n\}$ is the set of expert-agents;

$r_{i}$ is the project true assessment for the $i$-th expert;

$s_{i}$ is the assessment given by the $i$-th expert during the expert examination;

$s_{i} \in[d ; D]$, where $d$ and $D$, respectively, are the assessment lower and upper boundaries;

$x$ is the resulting expert examination assessment.

Let the resulting estimate be determined based on the $\pi(s)$ convolution function, as a continuous differentiable function with the following properties:

$$
\begin{array}{r}
\pi\left(\begin{array}{c}
s, \ldots, s \\
n
\end{array}\right)=s ; s \in[d ; D] . \\
\pi(s) \in\left[\min _{i \in N}\left\{s_{i}\right\} ; \max _{i \in N}\left\{s_{i}\right\}\right]
\end{array}
$$

For any $i \in N$ and $j \in N$, the following expressions are true: 


$$
\begin{gathered}
\pi\left(s_{1}, \ldots, s_{i}, \ldots, s_{j}, \ldots, s_{n}\right)=\pi\left(s_{1}, \ldots, s_{j}, \ldots, s_{i}, \ldots, s_{n}\right) \\
\frac{\partial \pi(s)}{\partial s_{i}}>0, i \in N
\end{gathered}
$$

\section{Expert interest in the results of expert examination}

Interest in the expert examination result corresponds to the fact that experts are interested in minimum discrepancy between their true project assessment and the resulting expert assessment.

The system consists of a Center organizing the expert examination and the expertplayers. In this case, the $i$-th expert target function could be represented as:

$$
f_{i}=\left[r_{i}-\pi(s)\right]^{2}, i \in N .
$$

Since any expert is interested in the minimum discrepancy between the true own assessment of the scientific and technical project and the resulting expert assessment, his/her goal consists of choosing a behavior strategy that would allow to minimize consequences (4).

Here, it is necessary, when analyzing a formal model, to find out primarily conditions for the Nash equilibrium situation existence, i.e. to determine the $S_{i}^{*}$ situation, where the following condition is satisfied:

$$
\left[r_{i}-\pi\left(s^{*}\right)\right]^{2}=\min _{z \in[d, D]}\left[r_{i}-\pi\left(s_{j \neq i}^{*}, z\right)\right]^{2}, i \in N
$$

Let us consider without losing generality, as in [1-3], that experts are arranged in the descending order of their true estimates:

$$
r_{1}>r_{2}>\ldots>r_{n}
$$

Statement 1. If the expert target function is determined as (4), the experts' true values satisfy condition (6), and condition (3) is valid for the expert assessment, then the Nash equilibrium situation exists, and it takes the following form:

$$
s_{1}^{*}=s_{2}^{*}=\ldots=s_{j-1}^{*}=D, s_{j}^{*}=w_{j}, s_{j+1}^{*}=\ldots=s_{n}^{*}=d .
$$

Moreover, $w_{j} \in[d ; D]$.

Proof. Let us determine the $j$ expert number, whose resulting expert assessment is the closest to his true project assessment from the following condition:

$$
\left|r_{j}-\pi(\underbrace{D, \ldots, D}_{j-1}, w_{j}, d, \ldots, d)\right|=\min _{k \in N}\left|r_{k}-\pi(\underbrace{D, \ldots, D}_{k-1}, w_{k}, d, \ldots, d)\right| j \in N .
$$

Conditions (6) ensure fulfillment of the following inequalities

$$
\left|r_{j}-\pi(\underbrace{D, \ldots, D}_{j-1}, w_{j}, d, \ldots, d)\right|<\left|r_{k}-\pi(\underbrace{D, \ldots, D}_{n-j+1}, w_{j}, d, \ldots, d)\right|, i=1,2, \ldots, j-1
$$

and 


$$
\left|r_{j}-\pi(\underbrace{D, \ldots, D}_{j-1}, w_{j}, d, \ldots, d)\right|<\left|r_{k}-\pi(\underbrace{D, \ldots, D}_{j-j+1}, w_{j}, \begin{array}{c}
d, \ldots, d \\
n-j+1
\end{array})\right|, k=j+1, j+2, \ldots, n
$$

Let us show that the $\{\underbrace{D, \ldots, D}_{j-1}, w_{j}, d_{n-j+1}, \ldots, d\}$ situation is the Nash equilibrium situation.

Indeed, it follows for the objective function of the j-th expert (4) from (5) and (7) that:

$$
\left[r_{j}-\pi(\underbrace{D, \ldots, D}_{j-1}, w_{j}, d, \ldots, d)\right]_{n-j}^{2}<\left[r_{i}-\pi(\underbrace{D, \ldots, D}_{j-1}, w_{j}, d, \ldots, d)\right]_{n-j}^{2}, i \neq j
$$

Accordingly, for the $i$-th expert $i=1,2, \ldots, j$-1 with $s_{i}<D$ due to condition (3), the following inequality is true:

$$
\left[r_{i}-\pi(\underbrace{D, \ldots, D}_{j-1}, w_{j}, d, \ldots, d)\right]_{n-j}^{2}<\left[r_{i}-\pi(\underbrace{D, \ldots, D}_{i-1}, s_{i}, \underbrace{D, \ldots, D}_{j-i}, w_{j}, d, \ldots, d)\right]_{n-j}^{2},
$$

and for the $k$-th expert $k=j+1, j+2, \ldots, n$ with $s_{k}>d$ also by virtue of condition (3), the following inequality is true:

$$
\left.\left[r_{k}-\pi(\underbrace{D, \ldots, D}_{j-1}, w_{j}, d, \ldots, d)\right]_{n-j}^{2}<\left[r_{k}-\pi(\underbrace{D, \ldots, D}_{j-1}, w_{j}, \underset{k-j-1}{d, \ldots, d, s_{k}, d, \ldots, d}]_{n-k}\right)\right]^{2} .
$$

Validity of inequalities (8) and (9) makes it possible to conclude that decrease in their expert assessment by the $i$-th experts, $i=1,2, \ldots, j-1$, and increase in their expert assessment by $k$-th experts, $k=j+1, j+2, \ldots, n$ leads only to an increase in the values of their target functions. And this means an even higher deviation of the resulting expert assessment from their true project assessment. Thus, expression presented above is the Nash equilibrium situation.

The statement is proven.

At the same time, issues should be considered not only related to analyzing the expert examination mechanism in the case, when experts are interested in increasing their ratings, but also related to a situation, when the team conducting expert examination includes both experts interested in the expert examination results and experts interested in improving their ratings.

\section{Project expert examination by two different groups of experts}

Groups of experts differ from each other facing that one group of experts is interested in achieving the resulting assessment close to their own, and the other experts are interested in increasing their ratings.

Thus, the $N=\{1,2, \ldots, n\}$ entire set of expert-agents could be represented as the $N=U \cup V$, where $U=\{1,2, \ldots, u\}$ is the set of expert-agents interested in achieving the resulting score close to their own, and $V=\{1,2, \ldots, v\}$ is a set of expert-agents interested in raising their ratings. Moreover, $u+v=n$. Works [1-3] determine for experts interested in increasing their rating target functions that reflect the expert's interest in obtaining the resulting expert assessment, as close as possible to the one reported by such an expert. Based on this and taking into account (4) the target function of experts included in a team conducting the expert examination, the following could be written down: 


$$
f_{i}=\left\{\begin{array}{ll}
{\left[r_{i}-\pi(s)\right]^{2},} & \text { для } i \in U \\
{\left[s_{i}-\pi(s)\right]^{2},} & \text { для } i \in V
\end{array} .\right.
$$

As well as above and without losing generality, let us assume that experts with the $i \in U$ numbers are arranged in the descending order of their true estimates:

$$
r_{1}>r_{2}>\ldots>r_{u} \text {. }
$$

Lemma 1. For any $s_{i} \in[d ; D], \quad i \in U$, there exists such $s_{i}=c \in[d ; D]$ that $c=\pi\left(s_{1}, s_{2}, \ldots, s_{u}, c, \ldots, c\right)$.

Proof. Let an arbitrary set $s_{i}^{\prime} \in[d ; D], i \in U$ be given. It follows from the $\pi(s)$ convolution function definition that $x=\pi\left(s_{1}^{\prime}, s_{2}^{\prime}, \ldots, s_{v}^{\prime}, c, \ldots, c\right)$. Let us define the $g$ function as follows:

$$
g(y)=\pi(s_{1}^{\prime}, s_{2}^{\prime}, \ldots, s_{u}^{\prime}, \underbrace{y, \ldots, y}_{v}) .
$$

Let us denote $g(d)=\pi(s_{1}^{\prime}, s_{2}^{\prime}, \ldots, s_{u}^{\prime}, \underbrace{d, \ldots, d}_{v})$, and $g(D)=\pi(s_{1}^{\prime}, s_{2}^{\prime}, \ldots, s_{u}^{\prime}, \underbrace{D, \ldots, D}_{v})$.

Moreover, if

$$
s_{1}^{\prime}=s_{2}^{\prime}=, \ldots,=s_{u}^{\prime}=d,
$$

then $g(d)=\pi(\underbrace{d, \ldots, d}_{n})=d$; and this corresponds to the fact that Lemma 1 is proved for set (12).

Similarly, for the set

$$
s_{1}^{\prime}=s_{2}^{\prime}=, \ldots,=s_{u}^{\prime}=D
$$

$g(D)=\pi(\underbrace{D, \ldots, D}_{n})=D$; and this corresponds to the fact that Lemma 1 is proved for set (13).

Now, let there be the following set:

$$
\left\{s_{1}^{\prime \prime}=s_{2}^{\prime \prime}=, \ldots,=s_{u}^{\prime \prime}\right\},
$$

where among all $j \in U$ there is always such a $q$ number that $s_{q}^{\prime \prime} \neq d, q \in U$. It follows from condition (3) that $g(d)>d$, and, similarly, there always such a $k$ number could be found that $s_{k}^{\prime \prime} \neq D, k \in U$, then $g(D)<D$.

Let us show that there exists such a $y^{*}$ value that makes the following equality true:

$$
g\left(y^{*}\right)=y^{*} \text {. }
$$


Let us denote: $f(y)=y-g(y), y \in[d ; D]$.

It is easy to see that for the set (14) $f(d)=d-g(d)<0$, and $f(D)=D-g(D)>0$.

It follows from the first Bolzano - Cauchy theorem that there is a $y^{*} \in(d, D)$ point, where the function value is equal to zero: $f\left(y^{*}\right)=0$. This means that equality (15) is satisfied, which, and, in turn, means that $c=\pi\left(s_{1}^{\prime \prime}, s_{2}^{\prime \prime}, \ldots, s_{u}^{\prime \prime}, c, \ldots, c\right)$ is true. The lemma is proved.

Let us note that the converse is also true.

Lemma 2. For any $s_{i}=c \in[d ; D], \quad i \in V$, there exist such $s_{i} \in[d ; D], \quad i \in U$ that $c=\pi\left(s_{1}, s_{2}, \ldots, s_{u}, c, \ldots, c\right)$.

Indeed, from (1) $\pi\left(\begin{array}{c}c, \ldots, c \\ n\end{array}\right)=c, c \in[d ; D]$. Let $\pi\left(\begin{array}{c}s_{1}, c, \ldots, c \\ n-1\end{array}\right)=S_{1}$. It follows from (3) that if $c<s_{1}$, then $c<S_{1}$, and if $c>s_{1}$, then $c>S_{1}$.

For definiteness, let us assume that $c>s_{1}$. The $f_{2}(s)$ function is defined as follows: $f_{2}(s)=\pi\left(s_{1}, s, c, \ldots, c\right), s \in[d ; D]$. It is easy to see that $f_{2}(d)=S_{2 d}<f_{2}(c)=S_{1}<c$, and $f_{2}(D)=S_{2 D}>f_{2}(c)>\pi\left(\begin{array}{c}c, \ldots, c \\ n\end{array}\right)=c$.

Thus, $f_{2}(s) \in\left[S_{2 d} ; S_{2 D}\right]$ and $c \in\left[S_{2 d} ; S_{2 D}\right]$.

It follows from the Bolzano-Cauchy theorem on intermediate values that there exists such a $s_{2} \in[d ; D]$ value, that $f_{2}\left(s_{2}\right)=c$. Thus, we get $\pi\left(s_{1}, s_{2}, c, \ldots, c\right)=c$.

Let us now define the $f_{3}(s)$ function as follows:

$$
f_{3}(s)=\pi\left(s_{1}, s_{2}, s, c, \ldots, c\right), s \in[d ; D] .
$$

It is easy to see that $f_{3}(d)=S_{3 d}<f_{3}(c)=c$ and $f_{3}(D)=S_{3 D}>f_{3}(c)>\pi(c, \ldots, c)=c$. Thus: $f_{3}(s) \in\left[S_{3 d} ; S_{3 D}\right]$ and $c \in\left[S_{3 d} ; S_{3 D}\right]$.

It follows from the Bolzano-Cauchy theorem on intermediate values that there exists such a $s_{3} \in[d ; D]$ value, that $f_{3}\left(s_{3}\right)=c$. Thus, we get $\pi\left(s_{1}, s_{2}, s_{3}, c, \ldots, c\right)=c$.

Carrying out similar reasoning for $(u-3)$ times, we obtain:

$$
\pi\left(s_{1}, \ldots, s_{u}, c, \ldots, c\right)=c .
$$

And since $n-u=v$, we get:

$$
c=\pi\left(s_{1}, s_{2}, \ldots, s_{u}, c_{v}, \ldots, c\right) .
$$

The lemma is proved. 
For target function (10), the $s_{i}^{*}$ Nash equilibrium situation is defined as follows

$$
\left\{\begin{array}{l}
{\left[r_{i}-\pi\left(s^{*}\right)\right]^{2}=\min _{z \in[d, D]}\left[r_{i}-\pi\left(s_{j \neq i}^{*}, z\right)\right]^{2}, i \in U} \\
{\left[s_{i}^{*}-\pi\left(s^{*}\right)\right]^{2}=\min _{z \in[d, D]}\left[z-\pi\left(s_{j \neq i}^{*}, z\right)\right]^{2}, i \in V}
\end{array}\right.
$$

Statement 2. If the experts' target functions are defined as (10), inequalities (11) are valid for true values of experts from the $U$ set, and condition (3) is valid for the expert assessment, then the Nash equilibrium situation exists, and it has the following form (17):

$$
\left\{s_{1}^{*}=s_{2}^{*}=\ldots=s_{j-1}^{*}=D, s_{j}^{*}=w_{j}, s_{j+1}^{*}=\ldots=s_{u}^{*}=d, \underset{v}{c, \ldots, c}\right\},
$$

where expert number $j$ is an expert, for whom the following condition is true:

$$
\left[r_{j}-\pi\left(s^{*}\right)\right]^{2}=\min _{z \in[d, D]}\left[c-\pi\left(s_{1}^{*}, \ldots, s_{j-1}^{*}, z, s_{j+1}^{*}, \ldots, s_{u}^{*}, c, \ldots, c\right)\right]^{2}, j \in U,
$$

and $w_{j} \in[d ; D]$.

Proof. It follows from Lemma 1 that for any $s_{i} \in[d ; D], i \in U$, there exists such $s_{i}=c \in[d ; D], i \in V$ that

$$
c=\pi\left(s_{1}, s_{2}, \ldots, s_{u}, c, \ldots, c\right)
$$

It means for the set

$$
\left\{s_{1}^{*}=s_{2}^{*}=\ldots=s_{j-1}^{*}=D, s_{j}^{*}=w_{j}, s_{j+1}^{*}=\ldots=s_{u}^{*}=d\right\}
$$

such $c$ could be found that

$$
\pi(\underbrace{D, \ldots, D}_{j-1}, w_{j}, \underbrace{d, \ldots, d}_{u-j}, c, \underset{v}{c}, c)=c .
$$

And this means that corresponding situation is the Nash equilibrium situation for experts with the $i \in V$ numbers.

Let us determine the $j$ expert number, whose resulting expert assessment is closest to his true project assessment from the following condition:

$$
\left|r_{j}-\pi\left(\begin{array}{c}
D, \ldots, D, w_{j}, d, \ldots, d, c, \ldots, c \\
j-1
\end{array}\right)\right|=\min _{k \in N}\left|r_{k}-\pi\left(\begin{array}{c}
D, \ldots, D, r_{k}, d, \ldots, d, c, \ldots, c \\
k-1
\end{array}\right)\right| .
$$

Conditions (11) ensure satisfaction of the following inequalities:

$$
\left|r_{j}-\pi(\underbrace{D, \ldots, D}_{j-1}, w_{j}, d, \ldots, d, c, \ldots, c)\right|<\left|r_{i}-\pi(\underbrace{D, \ldots, D}_{j-j+1}, w_{j}, \underbrace{d, \ldots, d, c, \ldots, c}_{u-j+1})\right|, i=1,2, \ldots, j-1
$$

and 


$$
\left|r_{j}-\pi(\underbrace{D, \ldots, D}_{j-1}, w_{j}, d, \ldots, d, c, \ldots, c)\right|<\left|r_{k}-\pi(\underbrace{D, \ldots, D}_{u-j+1}, w_{j}, d, \ldots, d, c, \ldots, c)\right|, k=j+1, j+2, \ldots, n
$$

Let us show that situation (17) is the Nash equilibrium situation for experts with the $j \in U$ numbers.

Indeed, it follows for target function of the $j$-th expert (10) from (16) and (18) that

$$
\left[r_{j}-\pi(\underbrace{D, \ldots, D}_{j-1}, w_{j}, d, \ldots, d, c, \ldots, c)\right]_{u-j}^{2}<\left[r_{i}-\pi(\underbrace{D, \ldots, D}_{j-1}, w_{j}, d, \ldots, d, c, \ldots, c)\right]_{u-j}^{2}, i \neq j .
$$

Accordingly, the following inequality is valid for the $i$-th expert $i=1,2, \ldots, j$-1 with $s_{i}<D$ due to condition:

$$
\left[r_{i}-\pi\left(\begin{array}{c}
D, \ldots, D, w_{j}, d, \ldots, d, c, \ldots, c \\
j-1
\end{array}\right)\right]^{2}<\left[r_{i}-\pi\left(\begin{array}{c}
D, \ldots, D, s_{i}, D, \ldots, D, w_{j}, d, \ldots, d, c, \ldots, c \\
i-1
\end{array} \underset{j-i}{u-j} \underset{v}{D-1}\right)\right]^{2},
$$

and for the $k$-th expert $k=j+1, j+2, \ldots, n$ with $s_{k}>d$ also by virtue of condition (11), the following inequality is valid:

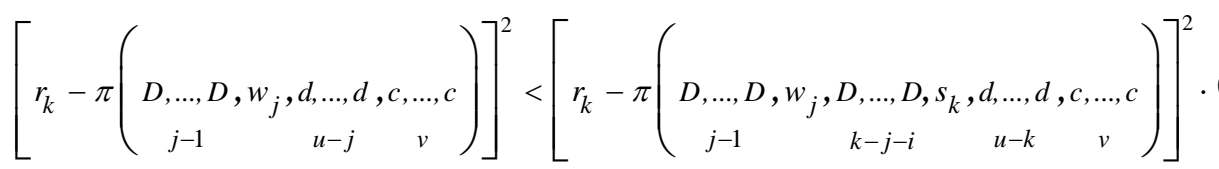

Validity of inequalities (19) and (20) makes it possible to conclude that decreasing in their expert assessment by the $i$-th experts $i=1,2, \ldots, j-1$ and increasing their expert assessment by the $k$-th experts $k=j+1, j+2, \ldots, n$ leads only to an increase in their target objective function values. And this means an even greater deviation of the resulting expert assessment from their true project assessment. Thus, expression (19) is the Nash equilibrium situation.

The statement is proven.

Let us assume here that the $\pi(s)$ convolution function is the power average of the agents' expert assessments. General view of the estimates' averaging is presented in the following form [4]:

$$
\pi\left(s_{1}, \ldots, s_{n}\right)=\left(\frac{\sum_{q=1}^{n} s_{q}^{b}}{n}\right)^{1 / b}=v\left(\sum_{q=1}^{n} s_{q}^{b}\right)^{1 / b}
$$

where $v=(1 / n)^{1 / b}$.

It was also shown in [4] that for various values of $b$, the most common average estimates are obtained:

The $b=1$ value corresponds to the arithmetic mean of all estimates:

$$
\pi(s)=\frac{1}{n} \sum_{i=1}^{n} s_{i}
$$

The $b=2$ value corresponds to the mean square of all estimates: 


$$
\pi(s)=\sqrt{\frac{\sum_{q=1}^{n} s_{q}^{2}}{n} .}
$$

The $b=-1$ value corresponds to the harmonic mean of all estimates:

$$
\pi(s)=\frac{n}{\sum_{i=1}^{n} \frac{1}{s_{i}}} .
$$

And finally, the $b \rightarrow 0$ value corresponds to the geometric mean of all estimates:

$$
\pi(s)=\left[\prod_{i=1}^{n} s_{i}\right]^{1 / n}
$$

For the case, when experts are seeking to obtain the resulting expert assessment value close to their own, it follows from the above provisions that for only one expert the resulting expert assessment could coincide with his true project assessment. And this coincidence is determined by the $\pi(s)$ averaging procedure of expert estimates.

Indeed, if there exists the $j$ number, for which $\pi(s)=r_{j}$, and $\pi(s)$ is represented in the form (21) for $\alpha_{q}=1, q=1, \ldots, n$, then the following could be written down:

$$
r_{j}=\left[\frac{(j-1) D^{b}+w_{j}^{b}+(n-j) d^{b}}{n}\right]^{1 / b} .
$$

Since $w_{j} \in[d ; D]$, then obviously the following inequalities are satisfied:

$$
\left[\frac{(j-1) D^{b}+(n+1-j) d^{b}}{n}\right]^{1 / b} \leq r_{j} \leq\left[\frac{j D^{b}+(n-j) d^{b}}{n}\right]^{1 / b} .
$$

To find this $j$ number, it is necessary to find $r_{j}$, for which (26) is valid. It is easy to see that, if for a certain $r_{j}$, inequality (26) is valid, then this corresponds to the $w_{j} \in[d ; D]$ existence, for which (7) is valid.

If inequality (26) is not satisfied for any $r_{i}, i \in N$, then this means that the Nash equilibrium situation has the form $\{\underbrace{D, \ldots, D}_{k}, d_{n-k}, \ldots, d\}$. And in this case, the task is to determine the $k$ number. This number is found from the following condition:

$$
\left|\left[\frac{(j-1) D^{b}+(n+1-j) d^{b}}{n}\right]^{1 / b}-r_{j}\right| \underset{j}{\longrightarrow} \min \text {. }
$$




\section{Conclusion}

The mechanisms of expert examination procedures considered in this article make it possible to evaluate complex projects with a high degree of correctness, including those connected to digitalization of scientific and technical products and optimization of resource management in the program implementation at universities.

At the same time, digitalization of scientific and educational activities of modern universities itself could produce an important consequence in the form of program elaboration for optimal management of their intellectual activity results. Solving the problems of increasing the efficiency of activities, educational institutions should use the potential of artificial intelligence, improve mechanisms for analytical processing of big data arrays and use new technologies being developed.

\section{References}

1. M.S. Agafonova, S.A. Barkalov, Conceptual Framework of Motivation and Stimulation in the Organization's Further Personnel Training, Modern European Researches, No. 4, pp. 25-30 (2017)

2. T.A. Averina, S.A. Barkalov, S.I. Moiseev, Application of the Theory of Latent Variables to Personnel Management Methods Society, Integration, Education / Proc. of the Int. Scientific Conf. (Rezekne), pp. 42-52 (2018) DOI:10.1088/1757899X/537/4/042048

3. T.V. Azarnova, S.A. Barkalov, V.V. Ukhlova, Estimation of Time Characteristics of Systems with Network Topology and Stochastic Processes of Functioning, Journal of Physics: Conference Series Materials of the International Conference "Applied Mathematics, Computational Science and Mechanics: Current Problems", Voronezh, 17-19 December 2018,Voronezh State University (2019)

4. S.A. Barkalov, V.N. Burkov, V.L. Poryadina, Models of the competitive mechanism at the organization of mechanical engineering production, IOP Conference Series: Materials Science and Engineering International Workshop "Advanced Technologies in Material Science, Mechanical and Automation Engineering - MIP: Engineering 2019", Krasnoyarsk Science and Technology City Hall of the Russian Union of Scientific and Engineering Associations, p. 42049 (2019)DOI: 10.1088/1757899X/537/4/042049

5. V.G. Borkovskaya, E. Degaev, I. Burkova, Environmental Economic Model of Risk Management and Costs in the Framework of the Quality Management System, MATEC Web of Conf., p. 19305027 (2018)DOI: 10.1051/matecconf/201819305027.

6. V. Burkov, M. Goubko, N. Korgin, D. Novikov, Introduction to Theory of Control in Organizations, Boca Raton: CRC Press, p. 332 (2015)

7. V. Burkov, D. Novikov, A. Shchepkin, Control Mechanisms for Ecological-Economic Systems Control, Berlin: Springer International Publishing, p. 174 (2015)

8. V.N. Burkov, I.V. Burkova, Ya.D. Gelrud, O.V. Loginovskiy, Smart Management Methods and Mechanisms of Industrial Enterprises and Organizations, Bulletin of the South Ural State University. Ser. Computer Technologies, Automatic Control, Radio Electronics,Vol. 16, No. 3, pp. 93-101 (2016). DOI: 10.14529/ctcr160310

9. V.N. Burkov, I.V. Burkova, K.E. Amelina, D.Y. Adamets, I.V. Goroshko, Management of complex project risks based on qualitative assessments, Proceedings of 
2018 11th International Conference "Management of Large-Scale System Development", MLSD (2018) C. 8551786 DOI:10.1109/mlsd.2018.8551786

10. V.N. Burkov, A.K. Enaleev, Optimal resource allocation in network structures, Proceedings of 2018 11th International Conference "Management of Large-Scale System Development", MLSD, p. 8551936 (2018)

11. V.N. Burkov, B.N. Korobets, V.A. Minaev, A.V. Shchepkin, Mechanisms of military and technological programs expert assessment, Herald of the Bauman Moscow State Technical University, Series Natural Sciences, No. 2 (71), pp. 105-117 (2017)

12. V.N. Burkov, O.V. Loginovskiy, O.I. Dranko, A.V. Hollay, The mechanisms of smart management for industrial enterprises, Applied Mathematics and Control Sciences, No. 1, pp. 59-73 (2020). DOI: 10.15593/2499-9873/2020.1.04.

13. I. Burkova, B. Titarenko, A. Hasnaout, R. Titarenko, Resource Allocation Problem in Project Management, 22nd International Scientific Conference on Construction the Formation of Living Environment, Tashkent, 18-21 April, pp. 1-12 (2019). DOI: 10.1051/e3sconf/20199701003

14. I.V. Burkova, A Method of Network Programming in Problems of Nonlinear Optimization, Automation and Remote Control, Vol. 70, No. 10, pp. 1606-1612 (2009). DOI: 10.1134/S0005117909100026.

15. I.V. Burkova, The network programming method in nonlinear optimization problems, Automation and Remote Control, No. 10, pp. 15-21 (2015). DOI: 10.1134/S0005117909100026

16. A.S. Dzhalolov, T.I. Buldakova, A. Proletarsky, Socio-economic decision support module by unstructured data, Proceedings of the 2020 IEEE Conference of Russian Young Researchers in Electrical and Electronic Engineering, EIConRus, p. 1931-1934 (2020)

17. S.A. Gudkova, T.S. Yakusheva, A.A. Sherstobitova, V.I. Burenina, Modeling of scientific intercultural communication of the teaching staff at smart university // Smart Innovation, Systems and Technologies, Vol. 144, pp. 551-560 (2019)

18. B.N. Korobets, Models for technology programs within an intellectual property management system, Herald of the Bauman Moscow State Technical University, Series Natural Sciences, No. 6 (69), pp. 135-142 (2016)

19. O.J. Kravets, S.A. Barkalov, N.A. Butyrina, V.D. Sekerin, A.E. Gorokhova, Processes of Multidimensional Classification of Scoring Objects with Heterogeneous Features Based on the Neural Networks Modeling

20. International Journal of Pure and Applied Mathematics, Vol. 119, No. 7a, pp. 875-879 (2018)

21. A.V. Proletarsky, K.A. Neusypin, K. Shen, N.A. Rachkovskaya, The complex innovation elaboration at the avionics engineering center, 2016 International Conference on Robotics and Automation Engineering, ICRAE 2016, pp. 48-52 (2016)

22. E. Turban, C. Pollard, G. Wood, Information technology for management: advancing sustainable, profitable business growth, John Wiley \& Sons, p. 386 (2015) 